\title{
LiteSense: An Adaptive Sensing Scheme for WSNs
}

\author{
João Marco C. Silva \\ HASLab, INESC TEC \\ Universidade do Minho \\ Email: joao.marco@inesctec.pt
}

\author{
Kalil Araujo Bispo \\ Departamento de Computação \\ Universidade Federal de Sergipe \\ Email:kalil@dcomp.ufs.br
}

\author{
Paulo Carvalho, Solange Rito Lima \\ Centro Algoritmi \\ Universidade do Minho \\ Email: \{pmc,solange\}@di.uminho.pt
}

\begin{abstract}
Adaptability and energy-efficient sensing are essential properties to sustain the easy deployment and lifetime of WSNs. These properties assume a stronger role in autonomous sensing environments where the application objectives or the parameters under measurement vary, and human intervention is not viable. In this context, this paper proposes LiteSense, a selfadaptive sampling scheme for WSNs, which aims at capturing accurately the behavior of the physical parameters of interest in each WSN context yet reducing the overhead in terms of sensing events and, consequently, the energy consumption. For this purpose, a set of low-complexity rules auto-regulates the sensing frequency depending on the observed parameter variation. Resorting to real environmental datasets, we provide statistical results showing the ability of LiteSense in reducing sensing activity and power consumption, while keeping the estimation accuracy of sensing events.
\end{abstract}

\section{INTRODUCTION}

WSNs may include several types of sensing, from continuous sensing to event detection or triggering of local actuators. This versatility allows their use in a wide spectrum of application fields, for instance, precision agriculture, intrusion detection and security systems, health care, wearables and environmental sensing. In many cases, WSNs operate without human intervention as devices substitution or maintenance may be impracticable, making the presence of self-management and power saving mechanisms strongly recommended [1]. In this way, sensor nodes must waste the minimum of power to accomplish their tasks, trying to maximize lifetime resorting to the utilization of efficient algorithms, lower-power usage components or reconfiguration procedures.

Considering that the energy consumed by the communication module when transmitting a bit across the network can overcome the energy required to process thousands of instructions [2], it is clear that reducing the volume of data transmitted is a key aspect in WSNs operation. Although this can be addressed resorting to data aggregation schemes, the underlying processes may imply data loss. Furthermore, the sensing subsystem is also a significant source of power consumption and, for this reason, sensing data should only be acquired when necessary, avoiding the waste of sensing, processing and transmission capacity.

In this context, this paper proposes LiteSense, an adaptive sampling scheme oriented to WSNs aiming at improving the trade-off between capturing data accurately and saving energy to enhance operational sensors' lifetime. The mechanism relies on self-regulation of sensing events in order to reduce the amount of data acquired and transmitted without human intervention. The proof-of-concept demonstrates that adaptive sampling can be a solid approach to reduce significantly the number of sensing events and power consumption, while maintaining an accurate view of WSN activity and behavior.

This paper is organized as follows: related work is discussed in Section II; the sampling scheme design goals and rational are described in Section III; the proof-of-concept and the corresponding evaluation results are discussed in Section IV; and the conclusions are summarized in Section V.

\section{RELATED WORK}

Although adaptive sampling has been successfully used in conventional computer networks [3], [4], the available solutions can hardly be applied in resource-constrained networks such as WSNs. Thus, several authors have devoted efforts in proposing adaptive sampling approaches for WSNs, attending to their inherent computational and energetic limitations.

In [5], it is proposed an optimal sensing scheduling policy for a power harvesting system equipped with a finite battery. This system aims to rule sensing frequency based on the battery power levels of sensor nodes, disregarding the variability of the observed parameter. In [6], it is proposed a recovering framework for big data sets through a small number of sensing events taking into account the space and time correlation properties from previous samples. The analysis resorts to probabilistic relations among variables involved in the compression, transmission and recovering processes. This process lowers the number of transmissions and transmission rate, disregarding the accuracy of the sensing process. Similarly, the authors in [7] establish a framework for collecting data from a WSN based on adaptive compressive sensing that considers the power consumption and the amount of information in sensing data. The study proposes an algorithm to obtain a more precise approximation of the measurements by wasting as less energy as possible. This proposal is also confined to adapt the transmission rate.

Taking into account the above discussion it is clear that most of the studies on adaptive sensing only cover a partial set of variables to optimise, such as the transmission rate, disregarding measurement accuracy. This evinces the need of studying and improving the trade-off between sensing events and accuracy for distinct WSN contexts. The present work intends to be a contribution in this respect. 


\section{LiteSENSE SAMPLING SCHEME}

The proposed sampling scheme aims at sensing physical parameters by detecting their values and variations accurately while saving sensor nodes' resources. The main design goals are identified in Figure 1 and explained next.

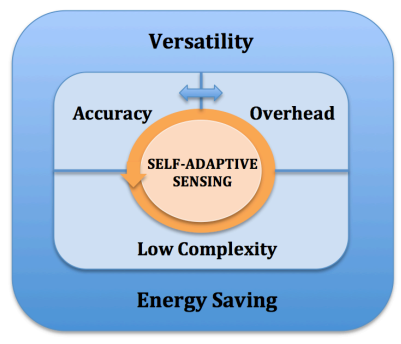

Fig. 1. LiteSense adaptive sampling - design goals

Versatility - the sampling scheme should be able to operate over distinct type of WSN measuring scenarios and parameters. This can be obtained endowing the sampling scheme with an intrinsic self-adaptability property in order to autoregulate the data sensing events according to each parameter behavior. It involves detecting variations on the measures and increasing/decreasing the sampling frequency accordingly.

Accuracy - the sampling scheme should be able to capture the correct behavior and values of the physical parameters under measurement. This involves identifying either the continuous behavior of a parameter being sensed or detecting critical and sporadic events.

Low overhead - the sampling process should reduce the overhead of sensing events, without compromising accuracy. This can be achieved taking advantage of parameter stability to decrease sampling frequency, reacting to variations with reduced impact on the overall performance.

Low complexity - the self-adaptive nature of the sampling scheme should be driven by simplicity of implementation and low consumption of resources. Adaptiveness should rely on a simple arithmetic process, e.g., inspired on TCP RTT estimation mechanism [8].

Energy saving - the sampling scheme should be able to monitor the parameters of interest based on energy-aware sensing, processing and communication subsystems. In this way, (i) reducing the number of sensing events needed to capture the parameter behavior; (ii) adapting the sampling frequency through low-cost algorithms; and (iii) reducing the number of transmissions to other sensors/actuators, are steps to impact positively on the energy consumption.

\section{A. Self-adaptive sampling}

As mentioned before, attending to the heterogeneity of application scenarios WSNs may have, self-adaptiveness is a mandatory property to assure. In this context, LiteSense adaptive sampling scheme uses the temporal variation in the observed scalar physical quantities in order to self-adjust the interval between two consecutive sensing events.
Basically, when the sampled values of the observed parameter do not vary significantly, the interval between two sensing events is increased, reducing its frequency, which leads to less computational effort and consequent less energy consumption [2]. Conversely, if a significant variation in the sampled parameter is observed, the time scheduling for the next sensing event is decreased in order to improve the accuracy in identifying its temporal fluctuation. Thus, when a sensing event is performed, the mean of the observed variable is calculated using the moving average $\bar{X}_{i}=(1-\alpha) \bar{X}_{i-1}+\alpha S$, where $\bar{X}_{i-1}$ is the mean calculated in the previous event and $\alpha$ is the weight of new observed value $S$.

Although the standard deviation $\sigma$ is the conventional choice for estimating the variance, it imposes costly operations to constrained devices. Alternatively, as discussed in [8], the mean deviation is a good approximation to $\sigma$, being easier to compute. Therefore, using the current mean value $\bar{X}_{i}$, the variation in the observed values is calculated resorting to the mean deviation $\bar{V}_{i}=(1-\beta) \bar{V}_{i-1}+\beta\left|\bar{X}_{i}-S\right|$, where, $\bar{V}_{i-1}$ is the mean deviation identified in the last sensing event and $\beta$ determines the weight of the current deviation $\left|\bar{X}_{i}-S\right|$.

As presented in Table I, the adaptive mechanism compares the current estimated mean variation $\left(\bar{V}_{i}\right)$ with the variation calculated in the previous sensing event $\left(\bar{V}_{i-1}\right)$ in order to identify if the observed parameter has changed significantly, and then, the time interval used to schedule the next sensing event is adjusted accordingly. If $\bar{V}_{i}$ is lower or equal to $\bar{V}_{i-1}$, the observed parameter did not change significantly from the last sensing events, which allows to reduce the sensing frequency by increasing $\Delta T$. Otherwise, the observed parameter changed significantly since the last observation, which requires more frequent sensing events, obtained by reducing $\Delta T$. An additional constraint is used to prevent $\Delta T$ from growing indefinitely $\left(\Delta T_{\max }\right)$, thus guaranteeing a minimum number of samples per time unit. Conversely, the maximum frequency of sampling $\left(\Delta T_{\min }\right)$ is also limited so that the interval between sensing events does not tend to zero, which would result in an overwhelming resource consumption. The factor $\epsilon$ in Table $\mathrm{I}$ is a filter expressing the scheme reactivity. This value may be set as a constant, indexed to the variation level of the observed parameter, or a function related to the battery level.

TABLE I

RULES TO SCHEDULE THE NEXT SENSING EVENT

\begin{tabular}{|c|c|}
\hline $\bar{X}_{i-1} \stackrel{\bar{V}_{i-1}}{\longleftrightarrow} \bar{X}_{i} \longleftrightarrow \bar{V}_{i} \longrightarrow S$ & $\Delta T_{i+1}$ \\
\hline \hline $\bar{V}_{i} \leq \bar{V}_{i-1}$ & $\min \left(\Delta T_{i}+\left(\Delta T_{i} \times \epsilon\right), \Delta T_{\max }\right)$ \\
\hline $\bar{V}_{i}>\bar{V}_{i-1}$ & $\max \left(\Delta T_{i}-\left(\Delta T_{i} \times \epsilon\right), \Delta T_{\min }\right)$ \\
\hline
\end{tabular}

\section{EVALUATION RESULTS}

This section provides the proof-of-concept for LiteSense, including a set of evaluation results regarding the ability of adaptive sampling in: (i) reducing the number of sensing events; (ii) identifying the temporal variation of the observed parameters accurately; (iii) self-adjusting the sensing 
frequency in accordance with the variability of environmental parameters; and (iv) reducing the power consumption of sensing and communication subsystems.

The analysis compares the performance of LiteSense adaptive sampling scheme to a deterministic scheme, widely used in monitoring scalar physical quantities. The publicly available dataset used in the tests was previously collected during approximately six hours, at intervals of five seconds, in a WSN using TelosB motes [9]. The WSN consists of eight sensors deployed in indoor and outdoor environments, sensing temperature (indoor: ST1,ST2; outdoor: ST3,ST4) and humidity (indoor: SH1,SH2; outdoor: $\mathrm{SH} 3, \mathrm{SH} 4)$. Aiming at evaluating the accuracy in identifying sudden changes in the observed parameters, sensors (ST1, ST4; SH1, SH4) were exposed to a steam of hot water to spark humidity and temperature levels.

The adaptive scheme was experimentally set with $\alpha=\beta=$ 0.7 in order to stress the weight of the latest sensed value and corresponding variation. The rules in Table I were set with $\epsilon=0.15$, meaning that the interval between samples $(\Delta T)$ is increased by a factor of $15 \%$ when the observed parameter remains stable, and reduced by the same factor when it changes significantly. As discussed in Section III-A, this adjustment may also be indexed to the observed variation or to the current battery level. In this proof-of-concept, $\Delta T_{\max }$ and $\Delta T_{\min }$ were set to 30 and 5 seconds, respectively.

The performance of LiteSense is evaluated relating the number of sensing events along the monitoring period with the accuracy in identifying the temporal variation of the observed parameters (temperature and humidity). As deterministic and adaptive sampling mechanisms yield to distinct number of samples, the statistical analysis considers the mean estimated value per second from the resultant time series. The accuracy is then estimated resorting both to the Mean Squared Error (MSE) and to the correlation between the time series.

\section{A. Sensing events and estimation accuracy}

Regarding the ability to reduce the number of sensing events, Figure 2 shows that, for all nodes and parameters, the adaptive scheme reduces in around $80 \%$ the number of events observed through the deterministic scheme. Attending to the lightweight algorithm ruling LiteSense, this significant reduction will also contribute to reduce energy consumption in the sensors, as discussed in Section IV-B.

However, despite of reducing the number of sensing events significantly, proving the efficiency of the proposed scheme requires verifying its ability to capture the real distribution of the observed parameters. This is accomplished by estimating the statistical representativeness of adaptive time series against the deterministic behavior. In this way, Figure 3 shows the distribution of sampled values along the test period, for indoor and outdoor sensors. The almost complete overlap of adaptive and deterministic resultant series, highlighted during both smooth and unstable periods, demonstrates that, even reducing the sensing events in around $80 \%$, the adaptive scheme in LiteSense has the capability to catch up the real parameter pattern. Note that, even in presence of sudden environmental changes

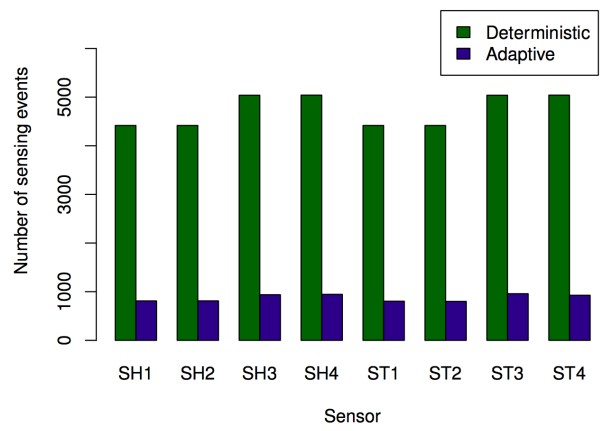

Fig. 2. Number of sensing events: Temperature (ST); Humidity (SH).
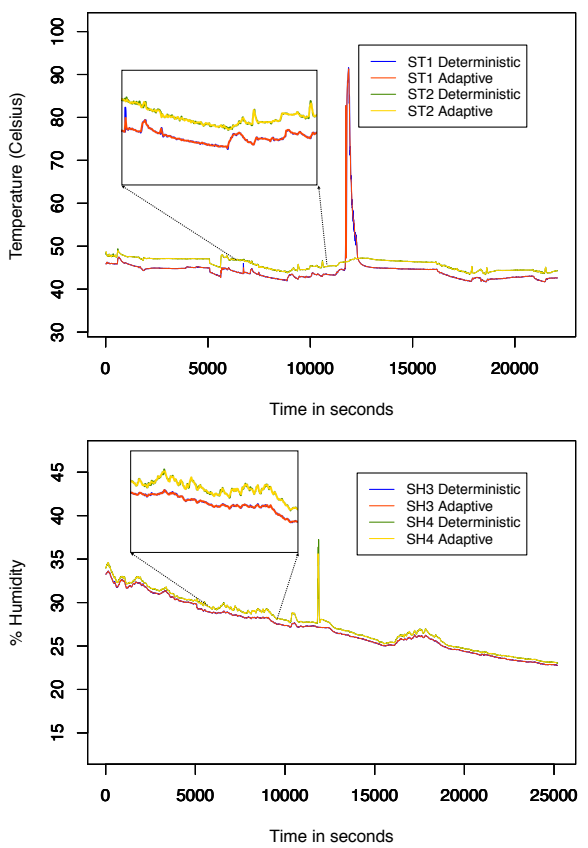

Fig. 3. Indoor observations (ST); Outdoor observations (SH).

affecting sensors ST1 and SH4, the adaptive scheme adjusts the sensing schedule correctly, confirming the versatility of LiteSense. These observations are corroborated through the high correlation ( 0.98 for all series) between the distributions of estimated parameters, and the low MSE (approximately 0.5 for temperature and 0.03 for humidity). This means that, for the considered scenarios, the estimation of humidity levels was more accurate, however, as depicted in Figure 3, the temperature was also accurately estimated.

The study of how the variation of ST1 impacts on the adaptiveness of $\Delta T$ along the time is illustrated in Figure 4. As shown, in presence of stable temperature readings (e.g., around $\mathrm{t}=15000$ seconds), $\Delta T$ assumes a steady behavior in its highest values, meaning that the sampling frequency is kept low. Conversely, upon more unstable periods of the sensed parameter (as in the anomaly peak mentioned previously), the mechanism reacts promptly reducing $\Delta T$, which is reflected in a sampling frequency increase. 


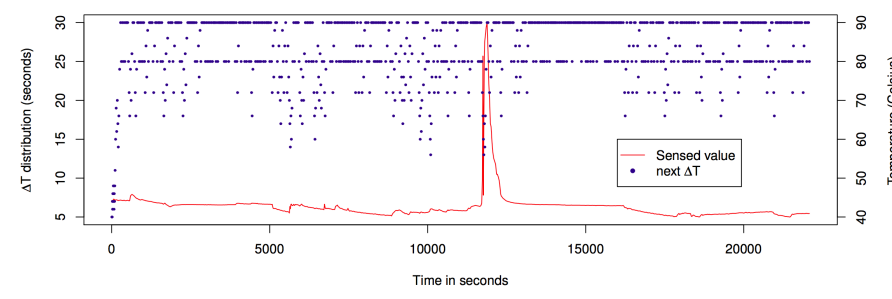

Fig. 4. ST1 - Distribution of $\Delta T$ regarding temperature variation.

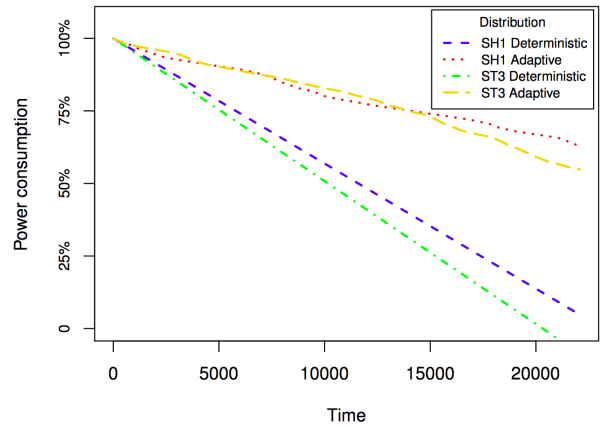

Fig. 5. Power Consumption: Indoor sensor (SH); Outdoor sensor (ST)

\section{B. Power Consumption}

Aiming at assessing the enhancement in power consumption promoted by LiteSense, the main operational states in the sensing and communication subsystems were identified and measured individually for each sensing event in a single-hop topology. The power consumption for different operation states in TelosB sensors is presented in Table II. The total consumption $\left(T_{P}\right)$ is reflected by the sum of partial average times in which the sensor was involved in transmitting, receiving, listening, sensing and sleeping events, i.e.,:

$$
\begin{array}{r}
T_{P}=T_{\text {transmitting }} \times n_{\text {tx }}+T_{\text {receiving }} \times n_{r x}+ \\
+T_{\text {sleeping }} \times n_{\text {sl }}+T_{\text {listening }} \times n_{l}+T_{\text {sensing }} \times n_{s}
\end{array}
$$

TABLE II

POWER CONSUMPTION FOR TELOSB OPERATIONAL STATES

\begin{tabular}{|c|c|}
\hline Operation state & Power consumption (W) \\
\hline \hline Transmitting & $5.9 E-5$ \\
\hline Receiving & $2.86 E-5$ \\
\hline Sleeping & $1.0 E-7$ \\
\hline Listening & $1.0 E-6$ \\
\hline Sensing & $0.5 E-6$ \\
\hline
\end{tabular}

Figure 5 shows the evolution of power consumption for sensors SH1 and ST3 along the performed tests. At the beginning, all sensors had batteries fully charged (i.e., 19160 $W$ ). As depicted, the progression of power consumed by the deterministic scheme is linear and leads to a faster battery drain when compared with the adaptive scheme. In this way, by using LiteSense, the power consumption is reduced in about $60 \%$ for all scenarios considered, which demonstrates that the ability in self-adapting sensing events has a major impact on reducing power consumption without affecting the accuracy of physical parameter measurements.

\section{CONCLUSIONS}

Attending to the inherent resource constraints of WSNs, this paper has proposed LiteSense, a self-adaptive sampling scheme aiming at capturing the behavior of multiple physical parameters accurately while reducing the overhead of sensing events and, consequently, the levels of energy consumption. The ability to adapt the sampling frequency through a low-cost algorithm was also defined as a design goal, due to the well known low-processing capabilities of sensor nodes. The proofof-concept has provided initial results attesting the proposal ability to measure environmental parameters accurately, while reducing in $80 \%$ the number of sensing events comparing to deterministic sampling. As future work, the tests will consider a wider range of sensing parameters, and the tuning of the proposed sampling scheme (e.g., exploring how $\epsilon$ can be related to both the variation observed in sensed values and the battery level) aiming at further increasing its versatility.

Acknowledgments - The research leading to these results has received funding from European Union's Horizon 2020 - The EU Framework Programme for Research and Innovation 2014-2020, under grant agreement No. 732505. This work has also been supported by COMPETE: POCI-010145-FEDER-007043 and FCT within the Project Scope: UID/CEC/00319/2013.

\section{REFERENCES}

[1] I. F. Akyildiz, W. Su, Y. Sankarasubramaniam, and E. Cayirci, "Wireless Sensor Networks: A Survey," Computer Networks: The International Journal of Computer and Telecommunications Networking, vol. 38, no. 4, pp. 393-422, 2002.

[2] G. Anastasi, M. Conti, M. Di Francesco, and A. Passarella, "Energy conservation in wireless sensor networks: A survey," Ad Hoc Networks, vol. 7, no. 3, pp. 537-568, 2009.

[3] E. A. Hernandez, M. C. Chidester, and A. D. George, "Adaptive Sampling for Network Management," Journal of Network and Systems Management, vol. 9, no. 4, pp. 409-434, 2001. [Online]. Available: http://dx.doi.org/10.1023/A:1012980307500

[4] J. M. C. Silva, P. Carvalho, and S. Rito Lima, "A multiadaptive sampling technique for cost-effective network measurements," Computer Networks, vol. 57, no. 17, pp. 3357-3369, Dec. 2013. [Online]. Available: http://dx.doi.org/10.1016/j.comnet.2013.07.023http: //linkinghub.elsevier.com/retrieve/pii/S1389128613002491

[5] J. Yang, X. Wu, and J. Wu, "Adaptive sensing scheduling for energy harvesting sensors with finite battery," in 2015 IEEE International Conference on Communications (ICC), June 2015, pp. 98-103.

[6] G. Quer, R. Masiero, G. Pillonetto, M. Rossi, and M. Zorzi, "Sensing, Compression, and Recovery for WSNs: Sparse Signal Modeling and Monitoring Framework," IEEE Transactions on Wireless Communications, vol. 11, no. 10, pp. 3447-3461, October 2012.

[7] C. T. Chou, R. Rana, and W. Hu, "Energy efficient information collection in wireless sensor networks using adaptive compressive sensing," in 2009 IEEE 34th Conference on Local Computer Networks, Oct 2009, pp. 443450

[8] V. Jacobson, "Congestion Avoidance and Control," in Symposium Proceedings on Communications Architectures and Protocols, ser. SIGCOMM '88. New York, NY, USA: ACM, 1988, pp. 314-329. [Online]. Available: http://doi.acm.org/10.1145/52324.52356

[9] S. Suthaharan, M. Alzahrani, S. Rajasegarar, C. Leckie, and M. Palaniswami, "Labelled data collection for anomaly detection in wireless sensor networks," in Intelligent sensors, sensor networks and information processing (ISSNIP), 2010 sixth international conference on. IEEE, 2010, pp. 269-274. 